MATHEMATICS OF COMPUTATION

Volume 71 , Number 237 , Pages $263-273$

S 0025-5718(01)01342-4

Article electronically published on October 4, 2001

\title{
COMPONENT-BY-COMPONENT CONSTRUCTION OF GOOD LATTICE RULES
}

\author{
I. H. SLOAN AND A. V. REZTSOV
}

\begin{abstract}
This paper provides a novel approach to the construction of good lattice rules for the integration of Korobov classes of periodic functions over the unit $s$-dimensional cube. Theorems are proved which justify the construction of good lattice rules one component at a time - that is, the lattice rule for dimension $s+1$ is obtained from the rule for dimension $s$ by searching over all possible choices of the $(s+1)$ th component, while keeping all the existing components unchanged. The construction, which goes against accepted wisdom, is illustrated by numerical examples. The construction is particularly useful if the components of the integrand are ordered, in the sense that the first component is more important than the second, and so on.
\end{abstract}

\section{INTRODUCTION}

Lattice rules (see [5, 99, [12]) are equal weight rules invented for the integration of periodic functions over the $s$-dimensional unit cube. While lattice rules vary greatly in their efficiency, theorems have been known for many years that assert the existence of "good" lattice rules. These are rules which in a certain sense integrate with guaranteed high accuracy periodic functions whose Fourier coefficients decay in a suitable way. The essence of such existence theorems is that they are not constructive; thus good rules are almost always found by searches (see for example [10, 3], 8], 7] and for other references 6]). The limitation of this approach is that the cost of an exhaustive search (for an $N$-point rule, with $N$ fixed) grows exponentially with the dimension $s$; thus in practice searches carried out for significant values of $s$ and $N$ have to be restricted in some way.

In this paper we present theoretical results which show that good lattice rules can be constructed one component at a time. The results may be thought surprising, since it is generally accepted that knowledge of a good lattice rule in $s$ dimensions does not help in finding a good rule in $s+1$ dimensions. Niederreiter (see p. 987 of [9]) expresses the prevailing view when he says "The task of finding good lattice points is complicated by the fact that their coordinates depend strongly on the dimensions. Thus, simplistic schemes such as taking a good lattice point $\left(g_{1}, \ldots, g_{s}\right)$ in dimension $s$ and searching for an integer $g_{s+1}$ so as to get a good lattice point $\left(g_{1}, \ldots, g_{s}, g_{s+1}\right)$ in dimension $s+1$ are, as a rule, doomed to failure."

Received by the editor May 5, 2000.

2000 Mathematics Subject Classification. Primary 65D30, 65D32.

(C)2001 American Mathematical Society 
Yet Theorem 2.1 below, which is the basic result of this paper, uses precisely the construction cautioned against in that quotation to construct lattice rules of guaranteed good quality in any number of dimensions.

In Section 2 the problem setting is established, and Theorem 2.1 stated. Theorem 2.1 is then proved in Section 3. In Section 4 we demonstrate that for $1 \leq s \leq s_{\max }$ (with $s_{\max } \geq 2$ chosen arbitrarily) the lattice rules constructed component by component through Theorem 2.1 are good lattice rules in the classical sense, with coefficients that depend on $s_{\max }$. The main result is Theorem 4.1.

In Section 5 we carry out numerical experiments based on Theorem 2.1. At the same time, for comparison we carry out searches based on the proposal by Korobov (see Chapter 3 of [5] or equation (6.90) of [6]) for limiting the search vectors $\mathbf{g}=\left(g_{1}, g_{2}, \ldots, g_{s}\right)$ to vectors of the form $\mathbf{g}=\left(1, g, g^{2}, \ldots, g^{s-1}\right)$ modulo $N$. The cost of the $s$-dimensional search in the Korobov case is $O\left(s N^{2}\right)$, or $O\left(s^{2} N^{2}\right)$ for the total search cost for all dimensions up to $s$. The cost of the latter is exactly the same as the cost of the algorithm based on the second part of Theorem 2.1. Generally the computed worst-case error is found to be smaller for the algorithm in Theorem 2.1 than for the Korobov construction when $s$ is large.

Lattice rules obtained by the present component-by-component algorithm may be particularly valuable if the user knows that for the integrand under consideration the first coordinate is more important than the second, the second than the third, and so on. This is because the error bounds in Theorems 2.1 and 4.1 hold simultaneously for all $s$ up to the dimension of the particular integral. Thus the error bounds hold for all the principal projections obtained by omitting one or more of the later coordinates of the integrand. This notion is successfully tested in Section 5 by applying the lattice rules to a simple product of 1-variable functions in which the successive factors become increasingly smooth.

\section{The SETting AND THE BASIC RESUlt}

The problem is to approximate the integral

$$
I f=\int_{[0,1]^{s}} f(\mathbf{x}) d \mathbf{x}=\int_{0}^{1} \ldots \int_{0}^{1} f\left(x_{1}, \ldots, x_{s}\right) d x_{1} \ldots d x_{s}
$$

by rank-1 lattice rules (see [9], 12]), that is, by rules of the form

$$
Q_{N}(\mathbf{g}) f=\frac{1}{N} \sum_{j=0}^{N-1} f\left(\left\{\frac{j}{N} \mathbf{g}\right\}\right)
$$

with $N>0$. Here $\mathbf{g}=\mathbf{g}^{(s)}$ is an $s$-dimensional integer vector that does not have $N$ as a factor, and by $\{\mathbf{x}\}=\left\{\left(x_{1}, \ldots, x_{s}\right)\right\}$ we denote the vector whose $j$ th component is the fractional part $\left\{x_{j}\right\}$ of $x_{j}$, for $j=1,2, \ldots, s$.

The function $f$ is assumed to belong to a Korobov class $E_{\alpha}^{(s)}$, defined as follows (see also [5], [12]): for $\alpha>1$ and $s=1,2,3, \ldots E_{\alpha}^{(s)}$ consists of all functions $f$ whose Fourier coefficients satisfy

$$
|\hat{f}(\mathbf{h})|=\left|\hat{f}\left(h_{1}, \ldots, h_{s}\right)\right| \leq \frac{1}{\left(\bar{h}_{1} \bar{h}_{2} \cdots \bar{h}_{s}\right)^{\alpha}},
$$

where for an arbitrary integer $h$

$$
\bar{h}:=\max (1,|h|) \text {. }
$$


It is well known that

$$
Q_{N}(\mathbf{g}) f-I f=\sum_{\substack{\mathbf{h} \in \mathbb{Z}^{s} \\ \mathbf{h} \cdot \mathbf{g} \equiv 0}}^{\prime} \hat{f}(\mathbf{h}),
$$

where the prime indicates that the term $\mathbf{h}=\mathbf{0}$ is to be omitted from the sum. Thus for $f \in E_{\alpha}^{(s)}$ the worst-case error is

$$
P_{\alpha, N}^{(s)}(\mathbf{g}):=\sum_{\substack{\mathbf{h} \in \mathbb{Z}^{s} \\ \mathbf{h} \cdot \mathbf{g} \equiv 0}}^{\prime} \frac{1}{\left(\bar{h}_{1} \bar{h}_{2} \cdots \bar{h}_{s}\right)^{\alpha}} .
$$

There is at least one member of $E_{\alpha}^{(s)}$ for which this worst-case error is achieved, namely, $f_{\alpha}^{(s)}$, where

$$
f_{\alpha}^{(s)}(\mathbf{x}):=\sum_{\mathbf{h} \in \mathbb{Z}^{s}} \frac{e^{2 \pi i \mathbf{h} \cdot \mathbf{x}}}{\left(\bar{h}_{1} \bar{h}_{2} \cdots \bar{h}_{s}\right)^{\alpha}},
$$

since from (5) and (6) we have

$$
P_{\alpha, N}^{(s)}(\mathbf{g})=Q_{N}(\mathbf{g}) f_{\alpha}^{(s)}-1 .
$$

For $\alpha$ an even integer $f_{\alpha}^{(s)}$ can be written explicitly as a product of polynomials of degree $\alpha$, making this an easy way of computing $P_{\alpha, N}^{(s)}(\mathbf{g})$. For example, for $\alpha=2$ it is known (see Section 4.2 of [12]) that

$$
f_{2}^{(s)}(\mathbf{x})=\prod_{j=1}^{s} F_{2}\left(x_{j}\right)
$$

where

$$
F_{2}(x)=1+2 \pi^{2}\left(x^{2}-x+\frac{1}{6}\right)
$$

The lattice rule (2) is clearly unchanged if to any component of $\mathbf{g}$ is added a multiple of $N$. Moreover, it is of little value to choose a component of $\mathbf{g}$ to be a multiple of $N$, since the corresponding coordinate of $\{j \mathbf{g} / N\}$ in that case always has the value zero. Thus it is sensible to choose each component of $\mathrm{g}$ from the restricted set

$$
\mathbf{Z}_{N}:=\{z \in \mathbb{Z} ; 1 \leq z \leq N-1\}
$$

Equivalently, we choose $\mathrm{g}$ from the tensor product

$$
\mathbf{Z}_{N}^{s}:=\underbrace{\mathbf{Z}_{N} \times \mathbf{Z}_{N} \cdots \times \mathbf{Z}_{N}}_{s \text { times }} .
$$

Korobov established (see Chapter 2 of [5]) that for each $s \geq 1$ and $\alpha>1$ and each prime number $N$ there exist $\mathbf{g} \in \mathbf{Z}_{N}^{s}$ and real numbers $D(s, \alpha)$ and $\eta(s, \alpha)$ such that

$$
P_{\alpha, N}^{(s)}(\mathbf{g}) \leq D(s, \alpha) \frac{(\log N)^{\eta(s, \alpha)}}{N^{\alpha}} .
$$

Sequences of vectors $\mathbf{g}=\mathbf{g}(N)$ for which (13) holds are traditionally called good lattice points. It is known (see [1], [9]) that sequences exist for which $\eta(s, \alpha) \leq$ $\alpha(s-1)$. On the other hand Sharygin [11] showed that $\eta(s, \alpha)$ is at least $s-1$. 
In the present work, as in [2], we establish bounds of the form (13) indirectly , by first obtaining, in the following theorem, bounds of the form

$$
P_{\alpha, N}^{(s)}(\mathbf{g}) \leq \frac{G(s, \alpha)}{N^{\alpha / \beta}}
$$

for arbitrary $\alpha \geq \beta>1$. We then deduce bounds of the form (13) in Section 4 .

The first part of the following theorem asserts, in effect, the existence of infinitedimensional lattice rules, all of whose principal projections have desirable error bounds. The second part of the theorem shows that rules of this kind can be constructed component by component.

As usual the zeta function is defined by

$$
\zeta(\beta):=\sum_{k=1}^{\infty} \frac{1}{k^{\beta}}
$$

for $\beta>1$.

Theorem 2.1. i) For arbitrary $\beta>1$ and prime $N$, with $N \geq 2 \zeta(\beta)+1$, there exists a sequence $\left(g_{j}\right)_{j=1}^{\infty}$, with $g_{j} \in \mathbf{Z}_{N}$, such that for all $s \geq 1$ and all $\alpha \geq \beta$

$$
P_{\alpha, N}^{(s)}\left(g_{1}, \ldots, g_{s}\right) \leq \frac{(1+2 \zeta(\beta))^{s \alpha / \beta}}{N^{\alpha / \beta}} .
$$

ii) The members $g_{j}$ of a sequence $\left(g_{j}\right)_{j=1}^{\infty}$ satisfying (16) can be determined recursively, by setting $g_{1}=1$ and taking $g_{s+1} \in \mathbf{Z}_{N}$ to be the least value of $g \in \mathbf{Z}_{N}$ that minimizes $P_{\beta, N}^{(s+1)}\left(g_{1}, \ldots, g_{s}, g\right)$.

\section{Proof of Theorem 2.1}

We first establish some notation. For $\beta>1$ and $\mathbf{W}$ a nonempty subset of $\mathbf{Z}_{N}^{s}$, we denote by $M_{\beta, N}^{(s)}(\mathbf{W})$ the mean of $P_{\beta, N}^{(s)}(\mathbf{g})$ over $\mathbf{g} \in \mathbf{W}$, that is,

$$
M_{\beta, N}^{(s)}(\mathbf{W}):=\frac{1}{|\mathbf{W}|} \sum_{\mathbf{g} \in \mathbf{W}} P_{\beta, N}^{(s)}(\mathbf{g}) .
$$

For the case $\mathbf{W}=\mathbf{Z}_{N}^{s}$ an explicit expression for this mean was obtained by Disney and Sloan [2] (see Section 4.5 of [12]), namely,

$$
M_{\beta, N}^{(s)}\left(\mathbf{Z}_{N}^{s}\right)=\frac{(1+2 \zeta(\beta))^{s}}{N}-1+\frac{N-1}{N}\left(1-2 \zeta(\beta) \frac{1-N^{1-\beta}}{N-1}\right)^{s} .
$$

In the next lemma we obtain a similar explicit expression for the mean over a smaller set: for any given $\mathbf{g}^{(s)}=\left(g_{1}, \ldots, g_{s}\right) \in \mathbf{Z}_{N}^{s}$ define $\mathbf{W}^{(s+1)}\left(\mathbf{g}^{(s)}\right) \subset \mathbf{Z}_{N}^{s+1}$ by

$$
\mathbf{W}^{(s+1)}\left(\mathbf{g}^{(s)}\right)=\mathbf{W}^{(s+1)}\left(g_{1}, \ldots, g_{s}\right):=\left\{\left(g_{1}, \ldots, g_{s}, g_{s+1}\right): g_{s+1} \in \mathbf{Z}_{N}\right\}
$$

a set of cardinality $\left|\mathbf{W}^{(s+1)}\left(\mathbf{g}^{(s)}\right)\right|=\left|\mathbf{Z}_{N}\right|=N-1$. For the mean over $\mathbf{W}^{(s+1)}\left(\mathbf{g}^{(s)}\right)$ we find: 
Lemma 3.1. Let $\beta>1, s \geq 1$, and $\mathbf{g}^{(s)} \in \mathbf{Z}_{N}^{s}$, and let $N$ be prime. Then

$$
\begin{aligned}
M_{\beta, N}^{(s+1)}( & \left.\mathbf{W}^{(s+1)}\left(\mathbf{g}^{(s)}\right)\right)=\frac{(1+2 \zeta(\beta))^{s+1}}{N}-1 \\
& \quad+\left(1-2 \zeta(\beta) \frac{1-N^{1-\beta}}{N-1}\right)\left(P_{\beta, N}^{(s)}\left(\mathbf{g}^{(s)}\right)-\frac{(1+2 \zeta(\beta))^{s}}{N}+1\right) .
\end{aligned}
$$

Proof. It follows from (8), on separating out the $j=0$ term in the quadrature sum (2), that

$$
\begin{aligned}
P_{\beta, N}^{(s)}\left(\mathbf{g}^{(s)}\right) & =\frac{1}{N} f_{\beta}^{(s)}(\mathbf{0})+\frac{1}{N} \sum_{j=1}^{N-1} f_{\beta}^{(s)}\left(\left\{\frac{j}{N} \mathbf{g}^{(s)}\right\}\right)-1 \\
& =\frac{(1+2 \zeta(\beta))^{s}}{N}-1+S_{\beta, N}^{(s)}\left(\mathbf{g}^{(s)}\right)
\end{aligned}
$$

where

$$
S_{\beta, N}^{(s)}(\mathbf{g}):=\frac{1}{N} \sum_{j=1}^{N-1} f_{\beta}^{(s)}\left(\left\{\frac{j}{N} \mathbf{g}\right\}\right) .
$$

Since the first two terms in (21) are independent of $\mathbf{g}^{(s)}$, it follows from (17) and (19), on replacing $s$ by $s+1$ and $\mathbf{g}$ by $\mathbf{g}^{(s+1)}=\left(\mathbf{g}^{(s)}, g_{s+1}\right)$ and then averaging over $g_{s+1}$, that

$$
\begin{aligned}
M_{\beta, N}^{(s+1)}\left(\mathbf{W}^{(s+1)}\left(\mathbf{g}^{(s)}\right)\right) & =\frac{(1+2 \zeta(\beta))^{s+1}}{N}-1 \\
& +\frac{1}{N-1} \sum_{g_{s+1} \in \mathbf{Z}_{N}} S_{\beta, N}^{(s+1)}\left(\left(\mathbf{g}^{(s)}, g_{s+1}\right)\right) .
\end{aligned}
$$

Now the last term can be written as

$$
\begin{aligned}
& \frac{1}{N-1} \sum_{g_{s+1}=1}^{N-1} \frac{1}{N} \sum_{j=1}^{N-1} f_{\beta}^{(s+1)}\left(\left\{\frac{j}{N}\left(\mathbf{g}^{(s)}, g_{s+1}\right)\right\}\right) \\
& =\frac{1}{N-1} \sum_{g_{s+1}=1}^{N-1} \frac{1}{N} \sum_{j=1}^{N-1} \sum_{\mathbf{h}^{(s)} \in \mathbb{Z}^{s}} \sum_{h_{s+1} \in \mathbb{Z}} \frac{e^{2 \pi i j \mathbf{h}^{(s)} \cdot \mathbf{g}^{(s)} / N}}{\left(\bar{h}_{1} \bar{h}_{2} \cdots \bar{h}_{s}\right)^{\beta}} \frac{e^{2 \pi i j h_{s+1} g_{s+1} / N}}{\bar{h}_{s+1}^{\beta}} \\
& =\frac{1}{N} \sum_{j=1}^{N-1} \sum_{\mathbf{h}^{(s)} \in \mathbb{Z}^{s}} \frac{e^{2 \pi i j \mathbf{h}^{(s)} \cdot \mathbf{g}^{(s)} / N}}{\left(\bar{h}_{1} \bar{h}_{2} \cdots \bar{h}_{s}\right)^{\beta}} T_{\beta, N}(j)
\end{aligned}
$$

where

$$
\begin{aligned}
T_{\beta, N}(j) & :=\frac{1}{N-1} \sum_{g_{s+1}=1}^{N-1} \sum_{h_{s+1}=-\infty}^{\infty} \frac{e^{2 \pi i j h_{s+1} g_{s+1} / N}}{\bar{h}_{s+1}^{\beta}} \\
& =\sum_{h=-\infty}^{\infty} \frac{1}{N-1} \sum_{g=1}^{N-1} \frac{e^{2 \pi i j h g / N}}{\bar{h}^{\beta}}
\end{aligned}
$$


On separating out the terms in which $h$ is a multiple of $N$ in the last expression we find, as in [2] (see also Section 4.5 of [12]), that for $j=1, \ldots, N-1$

$$
\begin{aligned}
T_{\beta, N}(j) & =\sum_{k=-\infty}^{\infty} \frac{1}{N-1} \sum_{g=1}^{N-1} \frac{1}{(\overline{N k})^{\beta}} \\
& +\sum_{\substack{h=-\infty \\
h \neq 0}}^{\infty} \frac{1}{\bar{h}^{\beta}} \frac{1}{N-1}\left(\sum_{g=0}^{N-1}\left(e^{2 \pi i j h / N}\right)^{g}-1\right) \\
& =\sum_{k=-\infty}^{\infty} \frac{1}{(\overline{N k})^{\beta}}-\sum_{h \neq 0}^{\infty} \bmod _{h=-\infty}^{\infty} \frac{1}{\bar{h}^{\beta}} \frac{1}{N-1} \\
& =\left(1+\frac{2 \zeta(\beta)}{N^{\beta}}\right)-\frac{1}{N-1}\left(1+2 \zeta(\beta)-1-\frac{2 \zeta(\beta)}{N^{\beta}}\right) \\
& =1-2 \zeta(\beta) \frac{1-N^{1-\beta}}{N-1},
\end{aligned}
$$

which, crucially for the remainder of the argument, is independent of $j$. Putting the results together, we find

$$
\begin{aligned}
M_{\beta, N}^{(s+1)}\left(\mathbf{W}^{(s+1)}\left(\mathbf{g}^{(s)}\right)\right) & =\frac{(1+2 \zeta(\beta))^{s+1}}{N}-1+T_{\beta, N}(1) \frac{1}{N} \sum_{j=1}^{N-1} f_{\beta}^{(s)}\left(\frac{j \mathbf{g}^{(s)}}{N}\right) \\
& =\frac{(1+2 \zeta(\beta))^{s+1}}{N}-1 \\
& +T_{\beta, N}(1)\left(Q_{N}\left(\mathbf{g}^{(s)}\right) f_{\beta}^{(s)}-\frac{1}{N} f_{\beta}^{(s)}(\mathbf{0})\right),
\end{aligned}
$$

which from (26), (8) and (7) is equivalent to the desired result.

From Lemma 3.1 follows easily:

Corollary 3.2. Let $\beta>1$ and $s \geq 1$, and let $N$ be a prime number satisfying $N \geq 2 \zeta(\beta)+1$. If $\mathbf{g}^{(s)} \in \mathbf{Z}_{N}^{s}$ is such that

$$
P_{\beta, N}^{(s)}\left(\mathbf{g}^{(s)}\right) \leq \frac{(1+2 \zeta(\beta))^{s}}{N}
$$

then there exists an integer $g_{s+1} \in \mathbf{Z}_{N}$ such that

$$
P_{\beta, N}^{(s+1)}\left(\mathbf{g}^{(s)}, g_{s+1}\right) \leq \frac{(1+2 \zeta(\beta))^{s+1}}{N} .
$$

Proof. Because $N \geq 2 \zeta(\beta)+1$, it follows easily that

$$
0 \leq 1-2 \zeta(\beta) \frac{1-N^{1-\beta}}{N-1} \leq 1
$$

Given the hypothesis (28), the result in Lemma 3.1 together with (30) yields the upper bound

$$
M_{\beta, N}^{(s+1)}\left(\mathbf{W}^{(s+1)}\left(\mathbf{g}^{(s)}\right)\right) \leq \frac{(1+2 \zeta(\beta))^{s+1}}{N}-1+1=\frac{(1+2 \zeta(\beta))^{s+1}}{N}
$$


Since $M_{\beta, N}^{(s+1)}\left(\mathbf{W}^{(s+1)}\left(\mathbf{g}^{(s)}\right)\right)$ is the mean of $P_{\beta, N}^{(s+1)}\left(\mathbf{g}^{(s)}, g_{s+1}\right)$ over $g_{s+1} \in \mathbf{Z}_{N}$ (see (17) and (19)), it follows that there must exist $g_{s+1} \in \mathbf{Z}_{N}$ such that

$$
P_{\beta, N}^{(s+1)}\left(\mathbf{g}^{(s)}, g_{s+1}\right) \leq M_{\beta, N}^{(s+1)}\left(\mathbf{W}^{(s+1)}\left(\mathbf{g}^{(s)}\right)\right) \leq \frac{(1+2 \zeta(\beta))^{s+1}}{N},
$$

proving the corollary.

Now we turn to the proof of Theorem 2.1. For $s=1, \beta>1$ and $g \in \mathbf{Z}_{N}$ it is easily seen from (6) that

$$
P_{\beta, N}^{(1)}(g)=\frac{2 \zeta(\beta)}{N^{\beta}} \leq \frac{1+2 \zeta(\beta)}{N} .
$$

Then Corollary 3.2 yields by induction

$$
P_{\beta, N}^{(s)}\left(\mathbf{g}^{(s)}\right) \leq \frac{(1+2 \zeta(\beta))^{s}}{N}, \quad s \geq 1,
$$

if the components of $\mathbf{g}^{(s)}$ are determined as in part $\left.i i\right)$ of the Theorem 2.1.

The inequality (34) gives the desired result in part $i$ ) of Theorem 2.1 for the case $\alpha=\beta$. To complete the proof of Theorem 2.1 it only remains to prove the bound (16) for $\alpha>\beta$. This follows immediately from (34) together with the next result.

Lemma 3.3. Let $\beta>1$, and let $N$ be a prime number. If $\mathbf{g}^{(s)} \in \mathbf{Z}_{N}^{s}$ and $\alpha \geq \beta$, then

$$
P_{\alpha, N}^{(s)}\left(\mathbf{g}^{(s)}\right) \leq\left(P_{\beta, N}^{(s)}\left(\mathbf{g}^{(s)}\right)\right)^{\alpha / \beta} .
$$

Proof. As noted in [12] (see the proof of Proposition 4.7), this follows from (6) together with Jensen's inequality (see [4], Theorem 19), which states that, for every sequence of positive numbers $\left(a_{j}\right)$,

$$
\left(\sum a_{j}^{p}\right)^{1 / p} \leq\left(\sum a_{j}^{q}\right)^{1 / q}
$$

if $0<q \leq p$.

The proof of Theorem 2.1 is now complete.

\section{Good Lattice points}

Because the parameter $\beta$ in Theorem 2.1 must exceed 1, it might be thought that the error bound from that theorem is inherently larger (and hence worse) than the bound (13) required for the method of good lattice points. In Theorem 4.1 we show that this is not so, by establishing that for any prescribed $\alpha>1$ and maximum dimension $s_{\max }$, the bound in Theorem 2.1 with an appropriate $\beta$ (fixed for the whole interval $1 \leq s \leq s_{\max }$ but depending on $N$ ) is bounded above by an expression of the good lattice point form (13). In other words, the sequence of vectors $\mathbf{g}^{(s)}=\left(g_{1}, \ldots, g_{s}\right)$, constructed one component at a time from Theorem 2.1 with the specified value of $\beta$, is a classical sequence of good lattice points in the space $E_{\alpha}^{(s)}$, for each $s=1, \ldots, s_{\max }$.

Theorem 4.1. i) Let $\alpha>1$ and let $s_{\max } \geq 2$ be a fixed positive integer, and let $N$ be a prime number satisfying

$$
N>e^{s_{\max } \frac{\alpha}{\alpha-1}} .
$$


There exists a finite sequence $\left(g_{j}\right)_{j=1}^{s_{\max }}$ such that for any $s$ satisfying $1 \leq s \leq$ $s_{\max }$

$$
P_{\alpha, N}^{(s)}\left(g_{1}, \ldots, g_{s}\right) \leq D(s, \alpha) \frac{(\log N)^{s \alpha}}{N^{\alpha}},
$$

where

$$
D(s, \alpha):=\left(\frac{3}{s_{\max }}\right)^{s \alpha} e^{s_{\max } \alpha} .
$$

ii) The sequence $\left(g_{j}\right)_{j=1}^{s_{\max }}$ can be constructed as in part i) of Theorem 2.1, with $\beta$ given by

$$
\beta:=\frac{\log N}{\log N-s_{\max }} .
$$

Proof. For each $N$ satisfying (37) there exists a unique $\beta$ such that $1<\beta<\alpha$ and

$$
N=e^{s_{\max } \frac{\beta}{\beta-1}},
$$

which is equivalent to (40).

The definition of the zeta function yields the bound

$$
\zeta(\beta)=\sum_{k=1}^{\infty} \frac{1}{k^{\beta}}<1+\int_{1}^{\infty} \frac{1}{x^{\beta}} d x=\frac{\beta}{\beta-1}=\frac{\log N}{s_{\max }} .
$$

From (42) and the fact that $e^{2 u}>2 u+1$ for $u>1$, we find that for $s_{\max } \geq 2$ there follows

$$
N>2 \zeta(\beta)+1
$$

Thus from Theorem 2.1 there exists a sequence $\left(g_{s}\right)_{s=1}^{\infty}$ satisfying (16) for all $s \geq 1$, and therefore in particular for $1 \leq s \leq s_{\max }$. It only remains to prove that the right-hand side of (16) is bounded by the right-hand side of (38) for $1 \leq s \leq s_{\max }$.

Now because $1 / \beta=1-(\beta-1) / \beta$, we can write, using (40),

$$
N^{-\alpha / \beta}=N^{-\alpha} e^{\alpha \log N\left(\frac{\beta-1}{\beta}\right)}=N^{-\alpha} e^{\alpha s_{\max }} .
$$

The next stage is to estimate $(1+2 \zeta(\beta))^{s \alpha / \beta}$. From $\zeta(\beta)>1$ together with $\beta>1$ and (42) we find

$$
(1+2 \zeta(\beta))^{s \alpha / \beta}<(3 \zeta(\beta))^{s \alpha}<\left(3 \frac{\log N}{s_{\max }}\right)^{s \alpha} .
$$

Collecting (44) and (45), we obtain from (16)

$$
P_{\alpha, N}^{(s)}\left(g_{1}, \ldots, g_{s}\right) \leq \frac{3^{s \alpha} e^{\alpha s_{\max }}(\log N)^{s \alpha}}{s_{\max }^{s \alpha} N^{\alpha}}, \quad s=1, \ldots, s_{\max },
$$

proving part $i$ ) of Theorem 4.1. Part $i i)$ of the theorem follows from the construction of the sequence $\left(g_{s}\right)_{s=1}^{\infty}$ used in the proof.

Remark 4.2. Notwithstanding the classical liking for inequalities of the form (38), the proof of Theorem 4.1 makes it clear that the right-hand side of (38) under the condition (37) is actually larger than the bound (16) with the same $s$ and $\beta$. It should also be recognised that the condition (37), which forces the number of points $N$ to grow exponentially with $s_{\max }$, makes Theorem 4.1 of limited practical applicability. 


\section{NUMERICAL EXPERIMENTS}

The (nonterminating) algorithm in the latter part of of Theorem 2.1 can be easily implemented for $\beta=2$ by using (8) with $\alpha$ replaced by $\beta=2$, because by (9) and (10) $f_{2}^{(s)}$ is available as a product of 1-dimensional quadratic functions; in practice it does not seem feasible to implement the algorithm for $\beta<2$.

For given $N$ and $\beta=2$, the precise algorithm is to compute successively $g_{1}, g_{2}, g_{3}, \ldots$, where $g_{s+1} \in \mathbf{Z}_{N}$ is the smallest minimizer of $P_{2, N}^{(s+1)}\left(g_{1}, \ldots, g_{s}, g\right)$ with respect to $g$.

In Table 1 we show for $N=1223$ the components $g_{s}$ computed by the above algorithm, together with the values of $P_{2, N}^{*}=P_{2, N}^{(s)}\left(g_{1}, \ldots, g_{s}\right)$. Also shown by comparison are the values of

$$
B_{2, N}^{*}:=\min \left\{P_{2, N}^{(s)}\left(1, k, k^{2}, \ldots, k^{s-1}\right): k=2, \ldots, N-1\right\}
$$

together with the minimizer $k$; for $s \leq 10$ these results agree with those obtained by Haber [3]. The quantities $M_{2, N}^{(s)}\left(\mathbf{Z}_{N}^{s}\right)$ in Table 1 are the averages of $P_{2, N}^{(s)}(\mathbf{g})$ over $\mathbf{Z}_{N}^{s}($ see (18)) .

Except for some relatively small values of $s$, the worst-case errors $P_{2, N}^{*}$ in Table 1 from the algorithm in Theorem 2.1 are generally somewhat smaller than

TABLE 1 . For $N=1223$, the numbers $g_{2}, g_{3}, \ldots$ are those found by the algorithm of Theorem 2.1 with $\beta=2$, and $P_{2, N}^{*}$ are the corresponding values of $P_{2, N}^{(s)}\left(1, g_{1}, g_{2}, \ldots, g_{s}\right)$. The optimal Korobov vectors (determined separately for each $s$ ) are $\left(1, k, k^{2}, \ldots, k^{s-1}\right)$ and the corresponding values of $P_{2, N}^{(s)}$ are denoted by $B_{2, N}^{*}$.

\begin{tabular}{|l|l|l|l|l|l|}
\hline$s$ & $g_{s}$ & $P_{2, N}^{*}$ & $B_{2, N}^{*}$ & $k$ & $M_{2, N}^{(s)}\left(\mathbf{Z}_{N}^{s}\right)$ \\
\hline 2 & 468 & $1.316 \mathrm{e}-4$ & $1.316 \mathrm{e}-4$ & 468 & $8.861 \mathrm{e}-3$ \\
3 & 263 & $4.837 \mathrm{e}-3$ & $4.520 \mathrm{e}-3$ & 377 & $5.569 \mathrm{e}-2$ \\
4 & 589 & $6.544 \mathrm{e}-2$ & $6.835 \mathrm{e}-2$ & 113 & $2.654 \mathrm{e}-1$ \\
5 & 18 & $5.923 \mathrm{e}-1$ & $5.734 \mathrm{e}-1$ & 69 & $1.174 \mathrm{e}+0$ \\
6 & 72 & $3.594 \mathrm{e}+0$ & $3.519 \mathrm{e}+0$ & 122 & $5.079 \mathrm{e}+0$ \\
7 & 108 & $1.786 \mathrm{e}+1$ & $1.805 \mathrm{e}+1$ & 25 & $2.184 \mathrm{e}+1$ \\
8 & 36 & $8.075 \mathrm{e}+1$ & $8.465 \mathrm{e}+1$ & 200 & $9.376 \mathrm{e}+1$ \\
9 & 36 & $3.509 \mathrm{e}+2$ & $3.810 \mathrm{e}+2$ & 202 & $4.023 \mathrm{e}+2$ \\
10 & 36 & $1.514 \mathrm{e}+3$ & $1.570 \mathrm{e}+3$ & 611 & $1.726 \mathrm{e}+3$ \\
11 & 36 & $6.524 \mathrm{e}+3$ & $7.170 \mathrm{e}+3$ & 35 & $7.404 \mathrm{e}+3$ \\
12 & 36 & $2.810 \mathrm{e}+4$ & $3.116 \mathrm{e}+4$ & 35 & $3.176 \mathrm{e}+4$ \\
13 & 36 & $1.210 \mathrm{e}+4$ & $1.348 \mathrm{e}+5$ & 35 & $1.363 \mathrm{e}+5$ \\
14 & 36 & $5.209 \mathrm{e}+5$ & $5.826 \mathrm{e}+5$ & 35 & $5.845 \mathrm{e}+5$ \\
15 & 36 & $2.242 \mathrm{e}+6$ & $2.504 \mathrm{e}+6$ & 35 & $2.507 \mathrm{e}+6$ \\
16 & 36 & $9.651 \mathrm{e}+6$ & $1.076 \mathrm{e}+7$ & 63 & $1.076 \mathrm{e}+7$ \\
17 & 36 & $4.154 \mathrm{e}+7$ & $4.614 \mathrm{e}+7$ & 35 & $4.614 \mathrm{e}+7$ \\
18 & 36 & $1.787 \mathrm{e}+8$ & $1.980 \mathrm{e}+8$ & 35 & $1.980 \mathrm{e}+8$ \\
19 & 36 & $7.689 \mathrm{e}+8$ & $8.492 \mathrm{e}+8$ & 268 & $8.492 \mathrm{e}+8$ \\
20 & 36 & $3.308 \mathrm{e}+9$ & $3.643 \mathrm{e}+9$ & 63 & $3.643 \mathrm{e}+9$ \\
\hline
\end{tabular}


the Korobov worst-case errors $B_{2, N}^{*}$, while for large $s$ the latter are similar to the means $M_{2, N}^{(s)}$.

An observation from Table 1 is that beyond $s=8$ the components of $\mathbf{g}$ found by the algorithm of Theorem 2.1 are unchanging. This is a seemingly characteristic feature seen also for other values of $N$.

The values of error in Table 1 for the larger values of $s$ are seen to be considerably bigger than 1. However it is well known, and confirmed by the next example, that this does not necessarily mean that the lattice rules themselves are not useful.

In the Introduction we speculated that lattice rules obtained by the algorithm of Theorem 2.1 may be particularly useful if the first component of $f$ is in some sense more important than the second, and so on. As our second experiment we test this idea by looking for the quadrature error for $F \in E_{2}^{(s)}$ given by the simple product

$$
F(\mathbf{x})=\prod_{j=1}^{s}\left(1+\frac{2 \pi^{2}}{j^{2}}\left(x^{2}-x+\frac{1}{6}\right)\right)
$$

in which the integral always equals 1 , but in the $j$ th factor $1+2 \pi^{2}\left(x^{2}-x+\frac{1}{6}\right) / j^{2}$ the second term (which expresses the departure from the mean value 1 ) is reduced by the factor $1 / j^{2}$ for $j=1, \ldots, s$ when compared to (9)-(10). In Table 2 we show the quadrature error $\left|Q_{N}\left(\mathbf{g}^{(s)}\right) F-I F\right|=\left|Q_{N}\left(\mathbf{g}^{(s)}\right) F-1\right|$, with $\mathbf{g}^{(s)}$ given by the second column of Table 1, and then for comparison also by the optimal Korobov vectors from Table 1.

The component-by-component results in the third column of Table 2 are indeed seen to be significantly better than the Korobov results for almost all values of

TABLE 2. Quadrature errors for the function $F$ defined by (48). Vectors $\mathbf{g}^{(s)}=\left(1, g_{2}, \ldots, g_{s}\right)$ and $\mathbf{g}_{k}^{(s)}=\left(1, k, \ldots, k^{s-1}\right)$ are given in Table 1.

\begin{tabular}{|l|l|l|l|l|}
\hline$s$ & $g_{s}$ & $P_{2, N}^{(s)}\left(\mathbf{g}^{(s)}\right)$ & $P_{2, N}^{(s)}\left(\mathbf{g}_{k}^{(s)}\right)$ & $k$ \\
\hline 2 & 468 & $3.455 \mathrm{e}-5$ & $3.455 \mathrm{e}-5$ & 468 \\
3 & 263 & $1.982 \mathrm{e}-4$ & $2.081 \mathrm{e}-4$ & 377 \\
4 & 589 & $5.148 \mathrm{e}-4$ & $6.407 \mathrm{e}-4$ & 113 \\
5 & 18 & $1.112 \mathrm{e}-3$ & $1.612 \mathrm{e}-3$ & 69 \\
6 & 72 & $1.966 \mathrm{e}-3$ & $2.979 \mathrm{e}-3$ & 122 \\
7 & 108 & $2.791 \mathrm{e}-3$ & $7.516 \mathrm{e}-3$ & 25 \\
8 & 36 & $3.946 \mathrm{e}-3$ & $6.627 \mathrm{e}-3$ & 200 \\
9 & 36 & $5.326 \mathrm{e}-3$ & $4.916 \mathrm{e}-2$ & 202 \\
10 & 36 & $6.750 \mathrm{e}-3$ & $2.089 \mathrm{e}-1$ & 611 \\
11 & 36 & $8.134 \mathrm{e}-3$ & $9.190 \mathrm{e}-2$ & 35 \\
12 & 36 & $9.443 \mathrm{e}-3$ & $9.241 \mathrm{e}-2$ & 35 \\
13 & 36 & $1.066 \mathrm{e}-2$ & $9.270 \mathrm{e}-2$ & 35 \\
14 & 36 & $1.180 \mathrm{e}-2$ & $9.284 \mathrm{e}-2$ & 35 \\
15 & 36 & $1.284 \mathrm{e}-2$ & $9.296 \mathrm{e}-2$ & 35 \\
16 & 36 & $1.381 \mathrm{e}-2$ & $8.555 \mathrm{e}-3$ & 63 \\
17 & 36 & $1.470 \mathrm{e}-2$ & $9.312 \mathrm{e}-2$ & 35 \\
18 & 36 & $1.552 \mathrm{e}-2$ & $9.319 \mathrm{e}-2$ & 35 \\
19 & 36 & $1.628 \mathrm{e}-2$ & $1.985 \mathrm{e}-2$ & 268 \\
20 & 36 & $1.699 \mathrm{e}-2$ & $8.964 \mathrm{e}-3$ & 63 \\
\hline
\end{tabular}


$s$. In some exceptional cases (e.g., $s=20$ ) the Korobov construction performs particularly well for the function $F$. It seems reasonable to regard such cases as accidental, given that neither algorithm has been optimised for the particular function $F$.

The technical report [13] includes in an appendix numerical results analogous to Table 1 for other prime numbers $N$, from $N=373$ to $N=7919$. The broad conclusions are similar to those above.

\section{ACKNOWLEDGMENTS}

The support of the Australian Research Council is gratefully acknowledged. So too are helpful comments by S. Joe and F. Kuo of Waikato.

\section{REFERENCES}

[1] Bahvalov, N.S. (1959) On approximate calculation of multiple integrals. (Russian) Vestnik Moskovskogo Universiteta. Seriya Matematiki, Mehaniki, etc. 4. pp. 3-18. MR 22:6077

[2] Disney, S.A.R. and Sloan, I.H. (1991) Error bounds for the method of good lattice points. Mathematics of Computation. 56. pp. 257-266. MR 91m:65068

[3] Haber, S. (1972) Experiments on optimal coefficients. In: Applications of number theory to numerical analysis (Ed S.K. Zaremba). pp. 11-37. Academic Press, New York. MR 52:12300

[4] Hardy, G.H., Littlewood, J.E. and Polya, G. (1934) Inequalities. Cambridge University Press, Cambridge.

[5] Korobov, N.M. (1963) Number-theoretical methods in approximate analysis. (Russian) Fizmatgiz, Moscow. MR 28:716

[6] Krommer, A.R. and Ueberhuber, C.W. (1998) Computational integration. SIAM, Philadelphia. MR 99g:65027

[7] Lyness, J.N. and Sorevik, T. (1991) A search program for finding optimal integration lattices. Computing. 47, pp. 103-120. MR 92k:65037

[8] Maisonneuve, D. (1972) Recherche et utilisation des "bons trellis". programmation et resultats numeriques. (French) In: Applications of number theory to numerical analysis (Ed S.K. Zaremba). pp. 121-201. Academic Press, New York. MR 49:8270

[9] Niederreiter, H. (1978) Quasi-Monte Carlo methods and pseudo-random numbers. Bulletin of the American Mathematical Society. 84. pp. 957-1041. MR 80d:65016

[10] Saltykov, A.I. (1963) Tables for computation of multiple integrals using the method of optimal coefficients. (Russian) Zhurnal Vychislitel'noi Matematiki i Matematicheskoi Fiziki. English translation: U.S.S.R. Computational Mathematics and Mathematical Physics. 3. pp. 235242. MR 27:962

[11] Sharygin. I.F. (1963) A lower estimate for the error of quadrature formulas for certain class of functions. (Russian) Zhurnal Vychislitel'noi Matematiki i Matematicheskoi Fiziki. English translation: U.S.S.R. Computational Mathematics and Mathematical Physics. 3. pp. 489497.

[12] Sloan, I.H. and Joe, S. (1994) Lattice methods for multiple integration. Clarendon Press, Oxford. MR 98a:65026

[13] Sloan, I.H. and Reztsov, A.V. (2000) Component-by-component construction of good lattice rules. Applied Mathematics Report AMR00/8, School of Mathematics, University of New South Wales. Also see WWW-version of this report at http://www.maths.unsw.edu.au/applied/reports/amr00.html.

School of Mathematics, University of New South Wales, Sydney, New South Wales 2052, Australia

E-mail address: i.sloan@unsw.edu.au

School of Mathematics, University of New South Wales, Sydney, New South Wales 2052, Australia 